\title{
Severe Pediatric Traumatic Brain Injury Treatment Approaches of Pediatric Intensivists in Turkey PICUs: National Survey Results
}

Nagehan Aslan ( $\square$ nagehan_aslan@hotmail.com )

Malatya Training and Research Hospital: Malatya Egitim ve Arastirma Hastanesi

https://orcid.org/0000-0002-6140-8873

\section{Dincer Yildizdas}

Cukurova University Faculty of Medicine: Cukurova Universitesi Tip Fakultesi, Cukurova University Faculty of Medicine: Cukurova Universitesi Tip Fakultesi

\section{Original research}

Keywords: Traumatic brain injury, pediatric, guideline, management

Posted Date: June 3rd, 2021

DOI: https://doi.org/10.21203/rs.3.rs-551276/v1

License: (c) (i) This work is licensed under a Creative Commons Attribution 4.0 International License. Read Full License 


\section{Abstract}

Objectives: Guidelines for the pediatric severe traumatic brain injury were published in 2003, 2012, 2019 in order to establish a common treatment approach. In this study, our aim is to evaluate the approaches of the pediatric intensive care specialists in Turkey, towards the follow-up and treatment of severe traumatic brain injury through a survey that was prepared in the light of the guidelines for the pediatric severe traumatic brain injury, which was updated by Kochanek et al.

Methods: The survey of forty-five questions on the SurveyMonkey was sent by e-mail to the centers, which were members of the Turkish Society of Pediatric Emergency and Intensive Care Medicine.

Results: A total of 45 centers participated in the survey. In all centers, first choice imaging method in the traumatic brain injury was the cranial brain tomography. Regarding the follow-up of the increased intracranial pressure, all centers reported that they used cranial brain tomography. In addition, 20 $(44.44 \%)$ centers mentioned using magnetic resonance imaging of the brain, 19 (42.22\%) centers mentioned using Near-Infrared Spectroscopy, 12 (26.67\%) centers mentioned using ultrasonographic optic nerve sheath diameter measurement, and 7 (15.56\%) centers mentioned using the transcranial Doppler ultrasonography. As the hyperosmolar therapy, 30 (65.22\%) centers reported using $3 \%$ hypertonic saline and $16(37.78 \%)$ reported using $3 \%$ hypertonic saline + mannitol. In rapid sequence intubation, the most common sedative drug used by the centers was midazolam. In addition, 40 (86.96\%) centers stated that they did not use prophylactic hyperventilation in the first 48 hours. In the first 7 days, prophylactic antiepileptic drugs were administered in 44 centers (97.78\%), and the most preferred antiepileptic drug was levetiracetam.

Conclusion: The results of our survey supported that the pediatric intensive care specialists in our country planned a large percentage of the treatment for pediatric patients with severe traumatic brain injury in line with the guidelines, which were updated in 2019. In patients with severe traumatic brain injury, it is possible to obtain the good long-term prognosis, prevent the secondary injury, and decrease the morbidity and mortality with the administration of appropriate treatments in the acute period.

\section{Introduction}

Head trauma is one of the most important causes of brain injury in children and can lead to mortality and lifelong physical, cognitive and behavioral morbidity. Like the differences in many clinical conditions between children and adults, childhood head traumas are also different from adults in many aspects (1). The possibility of head injury in the trauma is higher in children especially due to structural differences. In children, the possibility of the clinical condition to result in multiple organ failure and progression to shock is also high. For all these reasons, head traumas of the pediatric age group should be evaluated separately from adults (2).

Although there is a lack of pediatric data in the literature regarding traumatic brain injury (TBI) in children and newborns, a group of scientists published guidelines on pediatric severe head traumas in 2003 and 
then 2012. The same guidelines were updated in the light of recent studies in 2019. The latest pediatric severe head injury guidelines contain 22 suggestions in total. 3 of these suggestions are level II, and 19 are level III suggestions; there are no level I suggestions in the guidelines (3). As randomized controlled pediatric studies with large patient groups increase, it is hoped that stronger suggestions at the evidence level will be provided in the future. In this study, we aimed to evaluate the severe TBI and treatment approaches of pediatric intensive care professionals in the pediatric intensive care units in Turkey.

\section{Methods}

A multi-center, descriptive survey was prepared. The 45-question SurveyMonkey questionnaire we formed was sent to the member centers of the Turkish Society of Pediatric Emergency and Intensive Care Medicine by e-mail. Access to the questionnaire was enabled in July 2020. The questionnaire consisted of questions related to the capacity of the participating centers and units, and the approaches of the pediatric intensive care professionals working in the units to diagnosis, treatment and follow-up in critical pediatric patients with TBI. Approval was received from the XXXXX University Faculty of Medicine Clinical Research Ethics Committee for the study (Date:03/07/2020, meeting number:101). No statistical analysis methods were used. As the questions of the questionnaire did not contain any patient information, patient consent was not obtained.

\section{Results}

A total of 45 centers participated in the survey. Of the centers, 7 (15.56\%) were city hospitals, $12(26.67 \%)$ were state hospitals, 25 (55.56\%) were university hospitals, and 1 (2.22\%) was a private hospital. When the capacity of the centers was asked about, $23(51.11 \%)$ units were medical + surgical + cardiac intensive care, and $22(48.89 \%)$ units were medical + surgical intensive care. All the units were tertiary pediatric intensive care units. When we asked about the scoring they used for the neurological evaluation in the pediatric patient with traumatic brain injury, all of them expressed that they used the Glasgow Coma Scale (GCS), and 15 (33.33\%) centers additionally used the scoring 'Alert, Voice, Pain, Unresponsive' (AVPU). Forty-two of the centers (93.33\%) stated that they performed intracranial pressure (ICP) monitoring. In all the centers, the imaging method preferred first in TBI was cranial brain computed tomography (CT). Thirteen centers (28.89\%) stated that routine cranial CT was performed on the patient after 6 hours when the patient's cranial CT at the time of admission was normal and no increased ICP findings were observed in the patient's clinic. When the methods used to monitor the increase in ICP were questioned, as expressed by the centers, cranial CT was used by all the centers, brain magnetic resonance imaging (MRI) by 20 (44.44\%) centers, Near infrared spectroscopy (NIRS) by 19 (42.22\%) centers, bedside ultrasonographic optic nerve sheath diameter measurement (ONSD) by $12(26.67 \%)$ centers, and bedside transcranial Doppler ultrasonography (TCD) by $7(15.56 \%)$ centers. When the centers were asked about when they performed MRI in a patient with TBI in order to have information about secondary injury such as axonal injury, contusions, microhemorrhages and long-term prognosis, $1(2.22 \%)$ center stated that it 
performed it in the first 24 hours, $13(28.89 \%)$ centers in $24-72$ hours, and 31 (68.89\%) centers between 72 hours and 2 weeks.

In this patient group, the sedative drug used by the centers the most frequently in rapid sequential intubation (RSI) was midazolam with a rate of $97.78 \%$; the most common analgesic agent was fentanyl with a rate of $78.57 \%$, and the most frequent neuromuscular blocker agent was rocuronium with a rate of $71.79 \%$ (Graphic-1). When we questioned the hyperosmolar treatment approach, 30 (65.22\%) centers reported using $3 \%$ hypertonic saline (HS), and 16 (37.78\%) centers using $3 \% \mathrm{HS}+$ mannitol. With hyperosmolar treatment, the sodium value targeted by $22(47.8 \%)$ centers was $155-159 \mathrm{mEq} / \mathrm{L}$. The sodium treatment targets of the centers are given in Graphic-2. The most common side effect resulting from HS was natriuresis with a rate of $37.21 \%$ (Graphic-3). When the routine usage neuromuscular blocker agents were questioned in an intubated patient with TBI, 10 (21.74\%) centers expressed that they used routine neuromuscular blockers.

In the first 7 days, prophylactic antiepileptic drugs were administered in 44 centers (97.78\%), and the most frequently preferred antiepileptic drug was levetiracetam with a rate of $82.61 \%$. For this patient group, the target serum glucose was reported as $100-180 \mathrm{mg} / \mathrm{dL}$ by 28 centers $(60.87 \%)$. The number of the centers with the capability of continuous electroencephalogram (EEG) monitoring was 13 (28.26\%). $26(56.52 \%)$ centers expressed that they applied barbiturate therapy in addition to invasive arterial monitoring in patients with severe TBI who were hemodynamically stable. When the prophylactic hyperventilation approach was questioned in the first 48 hours, 40 (86.96\%) centers said that they did not apply prophylactic hyperventilation. When we questioned the target $\mathrm{PaCO}_{2}$ level in hyperventilation, as stated by the centers, 25 (55.55\%) centers targeted $30-35 \mathrm{mmHg}, 15(33.3 \%)$ centers $35-40 \mathrm{mmHg}$, and 5 centers $28-32 \mathrm{mmHg} \mathrm{PaCO}_{2}$. When we questioned the application of prophylactic moderate hypothermia $\left(32-33{ }^{\circ} \mathrm{C}\right), 39(86.67 \%)$ centers stated that they applied it. However, when we questioned their targeted body temperatures for this patient group, 19 (42.22\%) centers expressed that they only hindered hypothermia (Graphic-4). For endotracheal intubation, 23 (51.11\%) centers preferred the intravenous use of lidocaine. The number of the centers which performed decompressive craniectomy in intracranial hypertension resistant to medical treatment was $37(85.43 \%)$. The initiation time of enteral feeding was reported as the first 48 hours in $24(52.17 \%)$ centers.

\section{Discussion}

It is important to use a common terminology for evaluating consciousness to eliminate interpretation differences resulting from the knowledge and experience of the clinician. GCS, which is one of the consciousness evaluation tools developed for this purpose. The verbal and motor parts of the Glasgow Coma Scale value were modified for the evaluation of infants (4). AVPU can be used as another scale that will enable neurological evaluation. In a study evaluating the correlation between pediatric GCS and AVPU with a large pediatric series of 302 patients, a good correlation was discovered between the two scales, and it was found that the part of AVPU with the verbal response corresponds to 8 and above in GCS. Additionally, it was reported that AVPU should be used widely, especially in pre-hospital and 
emergency services, because it is a fast and easy scale (5). All the centers that participated in our survey stated that they used GCS, and 15 centers mentioned about using the AVPU scale in addition to GCS.

Cranial CT maintains its importance in the initial evaluation in TBI with its fastness, and superiority in evaluating the bone tissue and bleeding (6). lonizing radiation exposure is the most significant side effect of CT. Even though it was suggested in previous publications that cranial CT should be repeated 24-48 hours after the injury following a severe head trauma, according to the pediatric TBI guidelines renewed in 2019, it is not recommended to perform routine CT after 6 hours if the cranial CT is normal in the admission and there are no increased ICP findings in the patient's clinic. However, if the patient does not have neurological impairment or increased ICP findings, routine CT scans after 24 hours are not recommended for the decision of neurosurgical intervention (3). Accordingly, a decision should be made on the repetition of the brain CT according to the ICP and changes in the clinical examination. In our survey, in non-conformity with the suggestions of the guidelines, the number of the centers which stated that they performed routine cranial CT after 6 hours when the cranial CT of the patient was normal at admission and there were no increased ICP findings in the patient's clinic was $13(28.89 \%)$.

Brain MRI is not the first imaging option due to reasons such as long imaging time in head trauma cases, difficulty in monitoring during imaging, unsuitability of ventilator and infusion pumps for use during MRI, requirement of sedation, and high costs, but it may be of great significance with the information it provides about especially secondary injury, such as diffuse axonal injury, contusions and microhemorrhages, and long-term prognosis (7). In a survey study conducted with the participation of pediatric intensive care physicians, neurologists and neurosurgeons from a total of 27 centers from the United States, UK, Spain, Netherlands and India, 12 centers stated that they performed MRI on more than $95 \%$ of the patients with severe TBI. While $60 \%$ of the centers reported the timing of MRI between 72 hours and 2 weeks, only 1 center answered as the first 24 hours after trauma and 4 centers between 2472 hours (8). Similarly, in our survey, 31 (68.89\%) centers reported that they performed MRI between 72 hours and 14 days, $13(28.89 \%)$ centers in $24-72$ hours, and only $1(22.22 \%)$ center in the first 24 hours.

ICP monitoring with a ventricular catheter is accepted as the most accurate, cheap and reliable method, and meanwhile, the ability to perform cerebrospinal fluid drainage is a great advantage, because this is one of the implementations that can be performed in the first stage in the increased ICP treatment (9). ICP monitoring is recommended in patients with TBI, children with abnormality in the first brain CT and an initial GCS of 3-8 (3). In the pediatric TBI guideline updated in 2019, there is a suggestion of ICP monitoring as in the previous 2 published guidelines, and it is recommended to keep the ICP below 20 $\mathrm{mmHg}$ as the treatment goal (3). $93.33 \%$ of the centers that participated in our survey were observed to be able to perform ICP monitoring.

Near infrared spectroscopy is a noninvasive monitor which can detect regional brain tissue oxygenation, cerebral blood flow and cerebral blood volume changes, and provides continuous measurement (10). In our survey, 19 (42.22\%) centers stated that they used NIRS in the follow-up of ICP. 
In recent years, the use of point-of-care ultrasound by the clinicians who are not radiologists is becoming widespread (11). Optic nerve sheath is the continuation of the dura mater, arachnoid mater and pia mater. When the ICP increases, it is directly reflected on ONSD, and an increase is observed in ONSD even before the development of papillae stasis (12). In a pediatric study, Rehman Siddiqui et al. (13) reported the limit for ONSD as $>4 \mathrm{~mm}$ in the newborns, $>4.71 \mathrm{~mm}$ in those between $1-10$ years of age, and $>5.43$ in those above 10 years old (sensitivity $100 \%$, specificity $60-66.7 \%$ ). In a study on the evaluation of 72 pediatric patients in whom interventional ICP catheters were placed, the ONSD cut-off value was reported as 5.28 $\mathrm{mm}$ for ICP $>15 \mathrm{mmHg}$ and $5.57 \mathrm{~mm}$ for ICP $>20 \mathrm{mmHg}$ (14). Twelve (26.67\%) centers that participated in our survey were using ultrasonographic ONSD measurement in the follow-up of increased ICP.

In a review of the studies on the pediatric use of TCD in head traumas in the literature, it was suggested that TCD is a useful tool for evaluating autoregulation, vasospasm, and ICP after TBI (15). In our survey, the number of the centers using TCD in the clinical practice in a patient with increased ICP was 7 (15.56\%).

The brain injured by a severe head trauma is highly sensitive to hypoxia and hypotension, and these two extracerebral events increase mortality and morbidity (16). The first goal in treatment should be to enable and maintain normal oxygenation, ventilation and blood pressure. If the patient is unconscious, has signs of insufficient oxygenation-ventilation or seems in a shock-like state, the airway should be opened quickly and stabilized (17). Rapid sequence intubation should be applied if endotracheal intubation is required. In our survey, midazolam was found to be the most frequently used sedative agent, fentanyl to be the most common analgesic agent, and rocuronium to be the most used muscle relaxant for RSI. The drug selections of pediatric intensive care professionals in patients with TBI in Turkey are given in Graphic-1 in detail.

In a review of 101 adult and 55 pediatric studies on the use of ketamine and its effects on ICP in patients with traumatic brain injury, none of the studies reported that ICP increased during ketamine administration. However, when the studies in the review are examined in detail, it is observed that ketamine was administered together with midazolam (18). In a study evaluating the relationship between ketamine and ICP, it was expressed that there was not enough evidence that ketamine increased ICP apart from the patients with hydrocephalus, and it could be used in procedural sedation, analgesia and RSI (19). $91.11 \%$ of the centers participating in our survey reported that they did not use ketamine in RSI in the patient group with severe TBI.

Painful stimuli and stress lead to an increase in sympathetic tone and hypertension, and cerebral edema in the affected areas. Sedation and neuromuscular blockade are applied following necessary monitoring. Usually, it is preferred to use narcotic-based sedation, which will not affect the patient's hemodynamic condition (3). Endotracheal/intravenous lidocaine or intravenous thiopental can be used in ICP increases, which may occur during routine nursing care, and during endotracheal aspiration. In the last pediatric TBI guidelines, it is suggested that specific indications, selection and dosage of the analgesics, sedatives and neuromuscular blocking agents should be left to the clinician who provides the treatment (3). Moreover, it 
is recommended to avoid bolus midazolam and/or fentanyl bolus administrations due to the risk of cerebral hypoperfusion during ICP increase crises, together with the use of multiple treatments for increased ICP and appropriate routine sedation analgesia treatment. In our survey, the number of the centers that administered routine neuromuscular blockers in intubated patients with TBI was 10 (21.74\%), and the number of the centers that preferred the use of intravenous lidocaine for endotracheal aspiration and nursing care was $23(51.11 \%)$.

The importance of the osmolar load is of great importance in brain edema; therefore, osmolar treatment methods are included in the first stage treatment. While mannitol was preferred as the hypertonic agent in the past, the use of HS has come into prominence since the 1990s (20). In a study by Yildizdas et al. (21), where they evaluated the efficiency of HS and mannitol in 67 pediatric patients with brain edema, and the side effect profile, mortality was discovered to be significantly lower in the patient groups given HS. In the same study, hyperchloremic metabolic acidosis in 4 patients given HS and renal failure in 1 patient who received mannitol were reported. The authors suggested that HS was more effective and safer than mannitol in the treatment of cerebral edema. When we look at the suggestions of the latest pediatric TBI guidelines, it is recommended to apply $3 \% \mathrm{HS}$ solution, and $2-5 \mathrm{~mL} / \mathrm{kg} 10-20$ minutes (maximum 250 $\mathrm{ml}$ ) if there is an increase in ICP. The suggested goal in hypertonic saline treatment is to keep the ICP below $20 \mathrm{mmHg}(3)$. The dose of $3 \% \mathrm{HS}$ solution, which was $6.5-10 \mathrm{~mL} / \mathrm{kg}$ in the 2012 pediatric TBI guidelines, was changed to $2-5 \mathrm{~mL} / \mathrm{kg}$ in the final guidelines (3). Possible side effects resulting from HS include increased ICP with a rebound effect, renal failure, subarachnoid hemorrhage, natriuresis, dehydration, hyperchloremic acidosis, central pontine myelinosis and diabetes insipidus when treatment is discontinued (3). The most frequently reported side effect caused by HS in the questionnaire was natriuresis by $37.21 \%$ (Graphic-2).

Even though mannitol is widely used in the treatment of increased ICP in pediatric trauma patients, no studies have been found to be used as evidence or meet the inclusion criteria in the 2019 pediatric TBI guidelines. For this reason, there are no suggestions on mannitol in the guidelines although its wide use is known (3). When we questioned the hyperosmolar treatment approach of the centers participating in our survey, 30 (65.22\%) centers reported using $3 \%$ hypertonic saline (HS), and 16 (37.78\%) centers using $3 \%$ HS + mannitol. The sodium value targeted by $22(47.8 \%)$ centers with hyperosmolar treatment was 155-159 mEq/L (Graphic-3).

Studies demonstrate that the use of prophylactic antiepileptic drugs is protective against posttraumatic seizures (22). Intravenous phenytoin and levetiracetam are widely used in pediatric patients after a severe head trauma as prophylactic antiepileptic. In the last guideline there is a suggestion for the use of prophylactic anticonvulsants to prevent posttraumatic seizures, particularly in the first 7 days. The guidelines emphasize that there is currently insufficient evidence to suggest levetiracetam instead of phenytoin, in terms of both the effectiveness in preventing early posttraumatic seizures and the risk of toxicity (3). In the survey, prophylactic antiepileptic drugs were administered in 44 (97.78\%) centers within the first 7 days, and the most frequently preferred antiepileptic drug was levetiracetam by $82.61 \%$. The number of the centers with the capability of continuous EEG monitoring was $13(28.26 \%)$. 
The studies on adults showed that prophylactic hyperventilation after a severe head trauma causes neuronal death in the hippocampus and poor prognosis (23). Therefore, prophylactic hyperventilation should not be used and $\mathrm{PaCO}_{2}$ should be kept between $35-40 \mathrm{mmHg}$ in general. The latest guidelines suggest that prophylactic hyperventilation should be avoided by keeping the $\mathrm{PaCO}_{2}$ level under $30 \mathrm{mmHg}$ in the first 48 hours after the trauma. Hyperventilation with appropriate cerebral monitoring in the cases of refractory intracranial hypertension $\left(\mathrm{PaCO}_{2} 30-35 \mathrm{mmHg}\right.$ ) can be used as a method of treatment (3). In our survey, 40 (86.96\%) centers reported that they did not apply prophylactic hyperventilation approach for the $\mathrm{PaCO}_{2}$ level in the first 48 hours, in accordance with the suggestions of the guidelines. When we questioned the target $\mathrm{PaCO}_{2}$ level in hyperventilation, as stated by the centers, 25 (55.55\%) centers targeted $30-35 \mathrm{mmHg}, 15$ (33.3\%) centers $35-40 \mathrm{mmHg}$, and $5(11.11 \%)$ centers $28-32 \mathrm{mmHg} \mathrm{PaCO}_{2}$.

Experimental studies in animal models and clinical studies in children have put forward that hyperthermia is correlated with poor results, and prevention of hyperthermia after TBI in children has been recommended (24). In the light of the current literature, it is not recommended to apply prophylactic moderate hypothermia $\left(32-33^{\circ} \mathrm{C}\right)$ to improve the clinical results in the latest TBI guidelines; however, there is a suggestion about the mild hypothermia for ICP control (3). When we question prophylactic mild hypothermia $\left(32-33^{\circ} \mathrm{C}\right)$ in our survey, $39(86.67 \%)$ centers expressed that they did not apply it. When we questioned their targeted body temperatures for this patient group, they stated that $42.22 \%$ of the centers only hindered hyperthermia (Graphic-4).

Continuous EEG monitoring is necessary in cases where barbiturates are used in continuous intravenous infusion or at frequent intervals. Generally, pentobarbital or thiopental is used, and the dose of the drug is adjusted according to EEG and ICP. As the dose, the lowest dose keeping ICP under control is used (25). In the last TBI guidelines, high-dose barbiturate therapy is recommended in patients with refractory intracranial hypertension and stable hemodynamics despite maximum medical and surgical treatment. Barbiturates may cause hypotension since they may decrease cardiac output (26). The guidelines also include the suggestion that continuous invasive arterial blood pressure monitoring and cardiovascular support (to maintain adequate CPP) will be required (3). Our survey results revealed that $26(56.52 \%)$ centers could perform invasive arterial monitoring and apply barbiturate therapy in patients with severe TBI who were hemodynamically stable.

Decompressive craniectomy is a surgical treatment option in increased ICP. In the latest pediatric TBI guidelines published, decompressive craniectomy is suggested in cases of neurological deterioration and no response to medical treatment (3). It is necessary to be careful in patients undergoing decompressive craniectomy in respect of probable surgical complications, such as subdural hygroma, hydrocephalus, wound infection, hematoma or progression of an existing hematoma. In our survey, the number of the centers applying decompressive craniectomy in the increase in ICP resistant to medical treatment was 37 (85.43\%).

After a severe head trauma, close glucose monitoring should be carried out. In these patients, the serum glucose level should generally be kept between $100-200 \mathrm{mg} / \mathrm{dl}$ by using insulin infusion when necessary 
(27). When we questioned serum glucose targets for this patient group, $28(60.87 \%)$ centers stated the target of $100-180 \mathrm{mg} / \mathrm{dL}$.

The optimal calorie and nutritional content that should be given to pediatric patients who had severe head injury is not known yet. In the pediatric TBI guidelines, early enteral nutritional support (within 72 hours after injury) was suggested as it decreases mortality and improves the clinical course. Using an immune modulator diet is not recommended (3). In the centers participating in our survey, the time to start enteral nutrition was reported to be the first 48 hours in $24(52.17 \%)$ centers.

\section{Limitations}

The most important limitation of our study is the possibility that the results point at the opinions of the participants of the survey rather than what is applied, as in all voluntary surveys. Besides, the number of the participating centers remained below the expectations.

\section{Conclusion}

Consequently, regardless of the method or methods, the treatment of a child with severe head injury in the intensive care unit requires continuous and meticulous follow-up and a protocol regulated specifically to each intensive care unit's own conditions and in the light of current guidelines. The results of our survey support that pediatric intensive care professionals in our country plan treatment in pediatric patients with severe TBI at a high level according to the guidelines updated in 2019. In patients with severe TBI, it is possible to have a good long-term prognosis, prevent secondary injury, and decrease morbidity and mortality with appropriate treatments in the acute period.

\section{Abbreviations}

TBI: Traumatic brain injury

GCS: Glasgow coma scale

AVPU: Alert, Voice, Pain, Unresponsive

ICP: Intracranial pressure

CT: Computed tomography

MRI: Magnetic resonance imaging

NIRS: Near infrared spectroscopy

ONSD: Optic nerve sheath diameter measurement

TCD: Transcranial Doppler ultrasonography 
HS: Hypertonic saline

EEG: Electroencephalogram

RSI: Rapid sequential intubation

FDA: US Food and Drug Administration

CPP: Cerebral perfusion pressure

\section{Declarations}

\section{Ethics approval and consent to participate:}

Approval was received from the Faculty of Medicine Clinical Research Ethics Committee for the study. (Date:3/07/2020, Meeting number:101)

\section{Consent to publish:}

We have received informed consent from all subjects' parents or suitable proxies for publication of this paper and the written consent is held by our institution

\section{Availability of data and materials:}

All data generated or analysed during this study are included in this published article. The datasets used and/or analysed during the current study are available from the corresponding author on reasonable request.

\section{Competing interests:}

We have no conflicts of interest to disclose.

\section{Funding:}

The authors declare that they have no funding for the research reported.

\section{Authors' Contributions:}

NA analyzed and interpreted the data regarding the survey about the treatmant approaches of pediatric intensive care providers in patients with traumatic brain injury and increased intracranial pressure. The 
article written by NA and reviewed and edited by DY. All authors read and approved the final manuscript."

\section{Acknowledgements}

We want to thank the pediatric intensive care specialists in the participating units who were members of the Turkish Society of Pediatric Emergency and Intensive Care for their collaboration in our survey. We created a list of acknowledgement by their surnames:

Nihal Akçay, Emine Akkuzu, Başak Akyıldız, Fatih Akın, Nuri Alaçakır, Mehmet Alakaya, Ümit Altuğ, Ayşe Berna Anıl, Gazi Arslan, Illknur Arslan, Gürkan Atay, Ülkem Koçoğlu Barlas, Tolga Besci, Mehmet Çeleğen, Agop Çıtak, Yasemin Çoban, Demet Demirkol, Ener Çağrı Dinleyici, Muhterem Duyu, Serhat Emeksiz, Seher Erdoğan, Feyza İnceköy Girgin, Merve Havan, Fulya Kamit, Tanil Kendirli, Selman Kesici, Halil Keskin, Hasan Serdar Kıhtır, Arda Kılınç, Çelebi Kocaoğlu, Ökkeş Özgür Mart, Arzu Oto, Serhan Özcan, Serkan Özsoylu, Etem Pişkin, Leyla Telhan, Nazan Ülgen Tekerek, Özlem Temel, Mutlu Uysal, Muhammed Üdürgücü, Ayhan Yaman, Mutlu Uysal Yazıcı, Pınar Yazıcı, Osman Yeşilbaş, Filiz Yetimakman, Resul Yılmaz.

\section{References}

1. Kochanek PM. Pediatric traumatic brain injury: beyond the guidelines. Curr Treat Options Neurol 2005; 7: 441-50.

2. Adelson PD, Clyde B, Kochanek PM, Wisniewski SR, Marion DW, Yonas Cerebrovascular response in infants and young children following severe traumatic brain injury: a preliminary report. Pediatr Neurosurg 1997; 26: 200-7.

3. Kochanek PM, Tasker RC, Carney N, TottenAM, Adelson PD, Selden NR, et al. Guidelines For the Management of Pediatric Severe Traumatic Brain Injury, Third Edition: Update of the Brain Trauma Foundation Guidelines. Pediatr Crit Care Med 2019; 20: 1-82.

4. Reith FC, Van den Brande R, Synnot A, Gruen R, Maas Al. The reliability of the Glasgow Coma Scale: a systematic review. Intensive Care Med 2016; 42: 3-15.

5. Hoffmann F, Schmalhofer M, Lehner M, Zimatschek S, Grote V, Reiter K. Comparison of the AVPU Scale and the Pediatric GCS in Prehospital Setting. Prehosp Emerg Care 2016; 20: 493-8.

6. SarkarK, Keachie K, Nguyen UT, Muizelaar JP, Zwienenberg-Lee M, Shahlaie Computed tomography characteristics in pediatric versus adult traumatic brain injury. J Neurosurg Pediatr 2014; 13: 307-14.

7. Buttram SD, Garcia-Filion P, Miller J, Youssfi M, Brown SD, Dalton HJ, et al. Computed tomography vs magnetic resonance imaging for identifying acute lesions in pediatric traumatic brain injury. Hosp Pediatr 2015; 5: 79-84.

8. Ferrazzano PA, Rosario BL, Wisniewski SR, Shafi NI, Siefkes HM, Miles DK, et al. Use of magnetic resonance imaging in severe pediatric traumatic brain injury: assessment of current practice. J Neurosurg Pediatr 2019; 23: 471-9. 
9. Ma R, Rowland D, Judge A, Calisto A, Jayamohan J, Johnson D, et al. Complications following intracranial pressure monitoring in children: a 6-year single-center experience. $\mathrm{J}$ Neurosurg Pediatr 2018; 21: 278-83.

10. Mathieu F, Khellaf A, Ku JC, DonnellyJ, Thelin EP, Zeiler Continuous Near-Infrared Spectroscopy Monitoring in Adult Traumatic Brain Injury: A Systematic Review. J Neurosurg Anesthesiol $2019 \mathrm{Jul} 10$.

11. Volpicelli G, Elbarbary M, Blaivas M, Lichtenstein DA, Mathis G, Kirkpatrick AW, et al. International evidence-based recommendations for point-of-care lung ultrasound. Intensive Care Med 2012; 38: 577-91.

12. Shirodkar CG, Rao SM, Mutkule DP, HardeYR, Venkategowda PM, Mahesh Optic nerve sheath diameter as a marker for evaluation and prognostication of intracranial pressure in Indian patients: An observational study. Indian J Crit Care Med 2014; 18: 728-34.

13. Rehman Siddiqui NU, Haque A, Abbas Q, JurairH, Salam B, Sayani Ultrasonographic opic nerve sheath diameter measurement for raised intracranial pressure in a tertiary care center of a developing country. J Ayub Med Coll Abbottabad 2018; 30: 495-500.

14. Kerscher SR, Schöni D, Neunhoeffer F, WolffM, Haas-Lude K, Bevot A, et al. The relation of optic nerve sheath diameter (ONSD) and intracranial pressure (ICP) in pediatric neurosurgery practice- Part II: Influence of wakefulness, method of ICP measurement, intra-individual ONSD-ICP correlation and changes after therapy. Childs Nerv Syst 2020; 36: 107-15.

15. Köroğlu TF. Transcranial Doppler Sonography in the PICU. PediatrCrit Care Med 2020; 21: 99-100.

16. Kannan N, Wang J, Mink RB, Wainwright MS, Groner JI, Bell MJ, et al; PEGASUS (Pediatric Guideline Adherence Outcomes) Study. Timely Hemodynamic Resuscitation and Outcomes in Severe PediatricTraumatic Brain Injury: Preliminary Findings. Pediatr Emerg Care 2018; 34: 325-29.

17. Mtaweh H, Bell MJ. Management of pediatric traumatic brain injury. Curr Treat Options Neurol 2015; 17: 348.

18. Zeiler FA, Teitelbaum J, West M, Gillman The Ketamine Effect on ICP in Traumatic Brain Injury. Neurocritical Care 2014; 21: 163-73.

19. Green SM, Andolfatto G, Krauss BS. Ketamine and Intracranial Pressure: No Contraindication Except Hydrocephalus. Annals of Emergency Medicine 2015; 65: 52-4.

20. Fisher B, Thomas D, Peterson B: Hypertonic saline lowers raised intracranial pressure in children after head trauma. J Neurosurg Anesthesiol 1992; 4: 4-10.

21. Yildizdas D, Altunbasak S, Celik U, Herguner O. Hypertonic saline treatment in children with cerebral edema. Indian Pediatr 2006; 43: 771-9.

22. Tanaka T, Litofsky NS. Anti-epileptic drugs in pediatric traumatic brain injury. Expert Rev Neurother 2016; 16: 1229-34.

23. Skippen P, Seear M, Poskitt K, Kestle J, Cochrane D, Annich G, et al. Effect of hyperventilation on regional cerebral blood flow in head-injured children. Crit Care Med 1997; 25: 1402-9. 
24. Adelson PD, Ragheb J, Kanev P, Brockmeyer D, Beers SR, Brown SD, et al. Phase II clinical trial of moderate hypothermia after severe traumatic brain injury in children. Neurosurgery 2005; 56: 740-54.

25. Mellion SA, Bennett KS, Ellsworth GL, Moore K, Riva-Cambrin J, Metzger RR, et al. High-dose barbiturates for refractory intracranial hypertension in children with severe traumatic brain injury. Pediatr Crit Care Med 2013; 14: 239-47.

26. Majdan M, Mauritz W, Wilbacher I, Brazinova A, Rusnak M, Leitgeb J. Barbiturates use and its effects in patients with severe traumatic brain injuryin five European countries. J Neurotrauma 2013; 30: 239.

27. Beca J, McSharry B, Erickson S, Yung M, Schibler A, Slater A, et al; Pediatric Study Group of the Australia and New Zealand Intensive Care Society Clinical Trials Group: Hypothermia for traumatic brain injury in children-a phase ii randomized controlled trial. Crit Care Med 2015; 43: 1458-66.

\section{Figures}




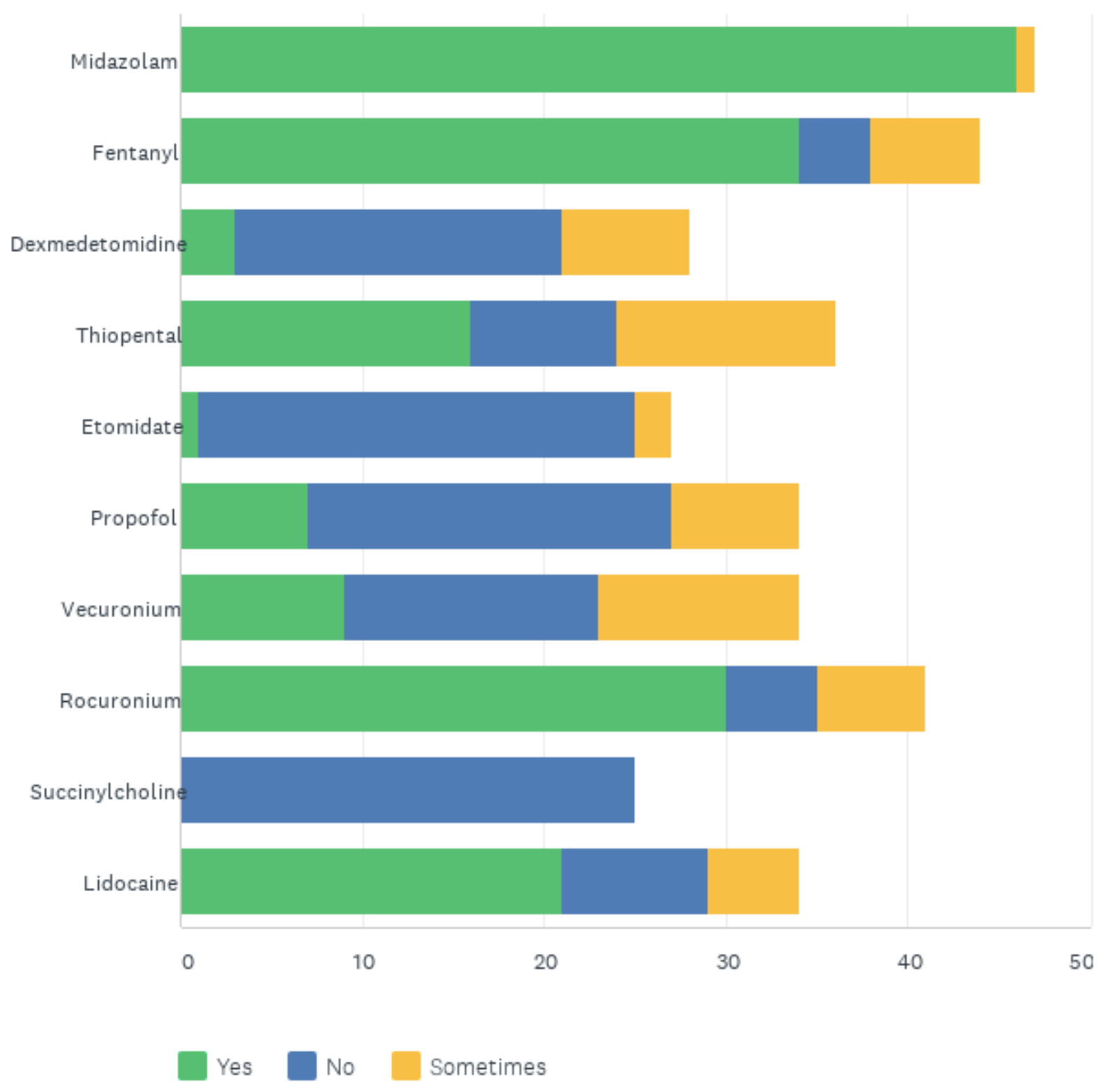

Figure 1

Graphic-1: Sedative and analgesic drug choices for rapid sequence intubation used by participant pediatric intensivists 


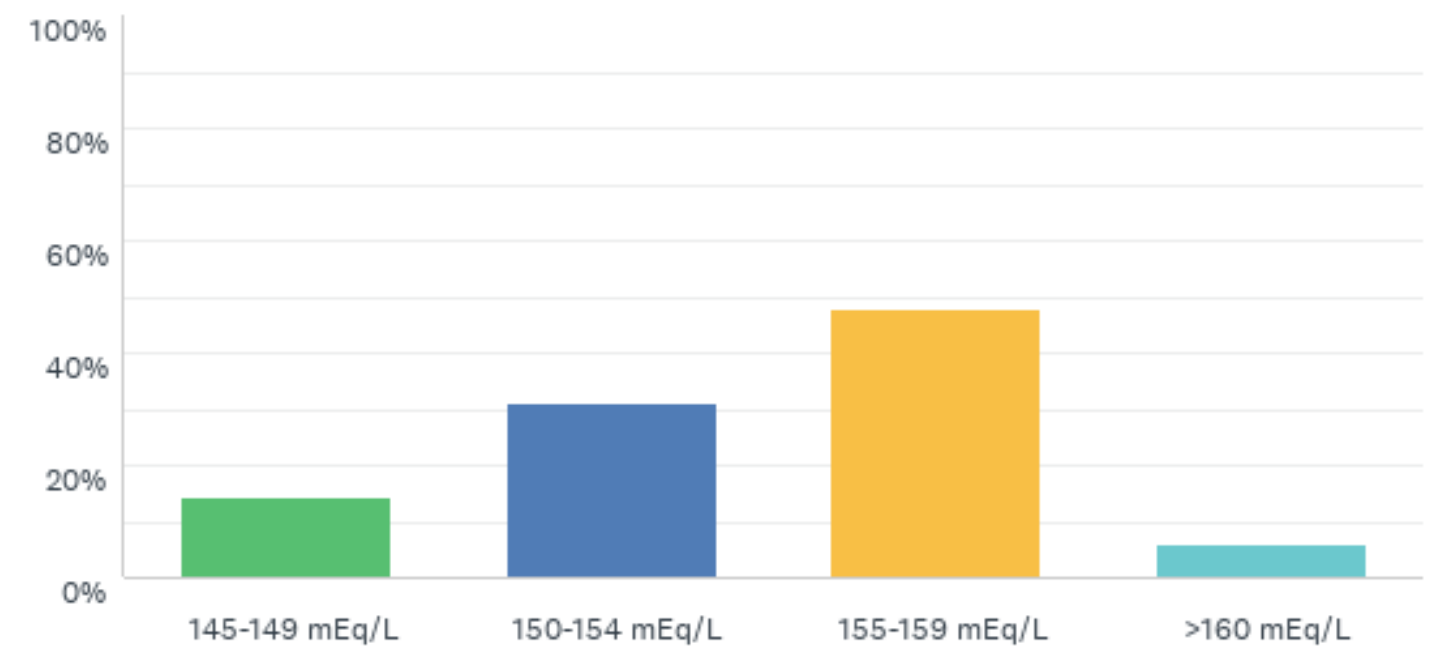

\section{Figure 2}

Graphic-2: Target serum sodium levels with hyperosmolar treatment in pediatric TBI patients for participant pediatric intensivists 


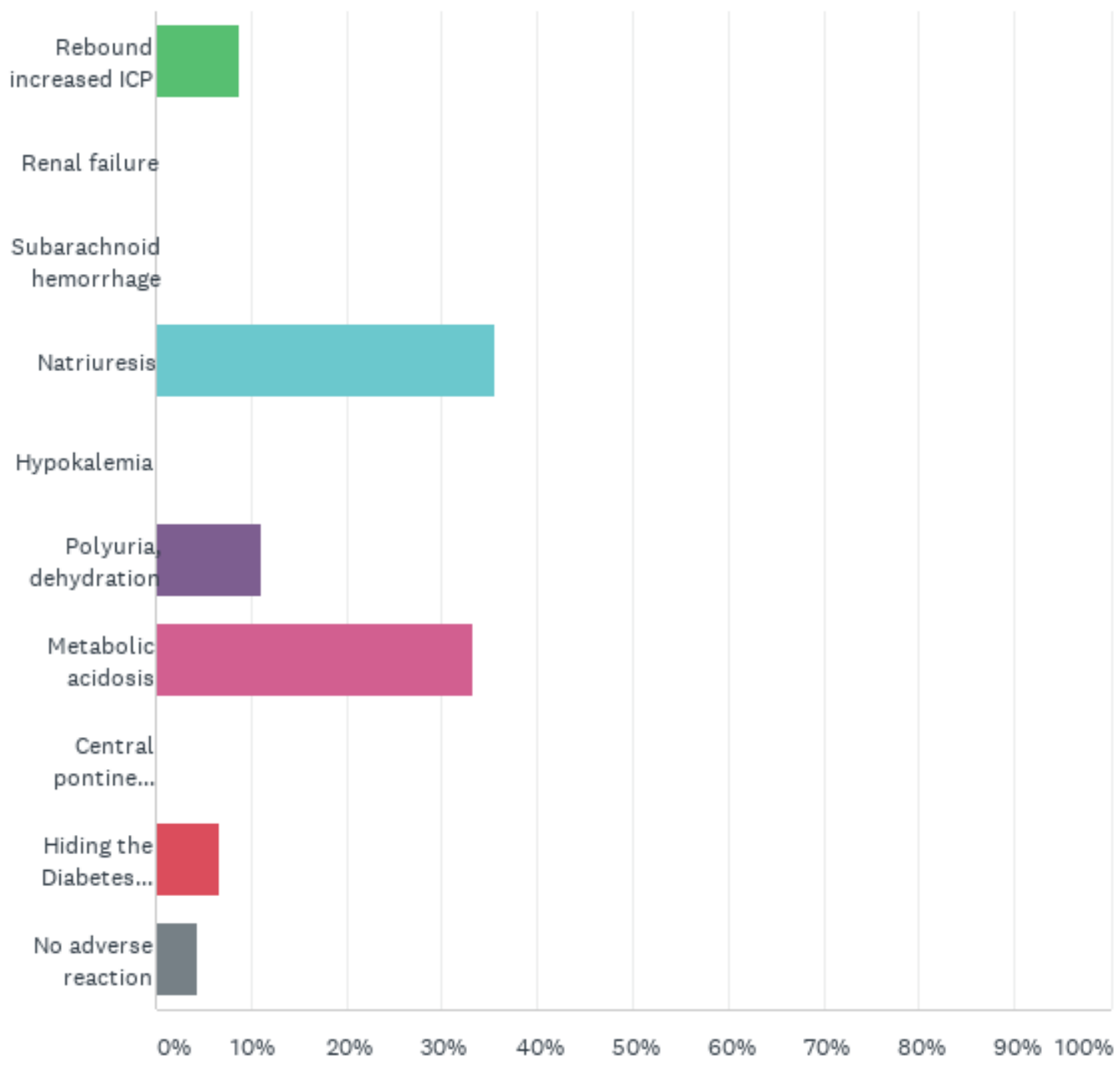

Figure 3

Graphic-3: Side effects due to hypertonic saline treatment reported by participant pediatric intensivists 


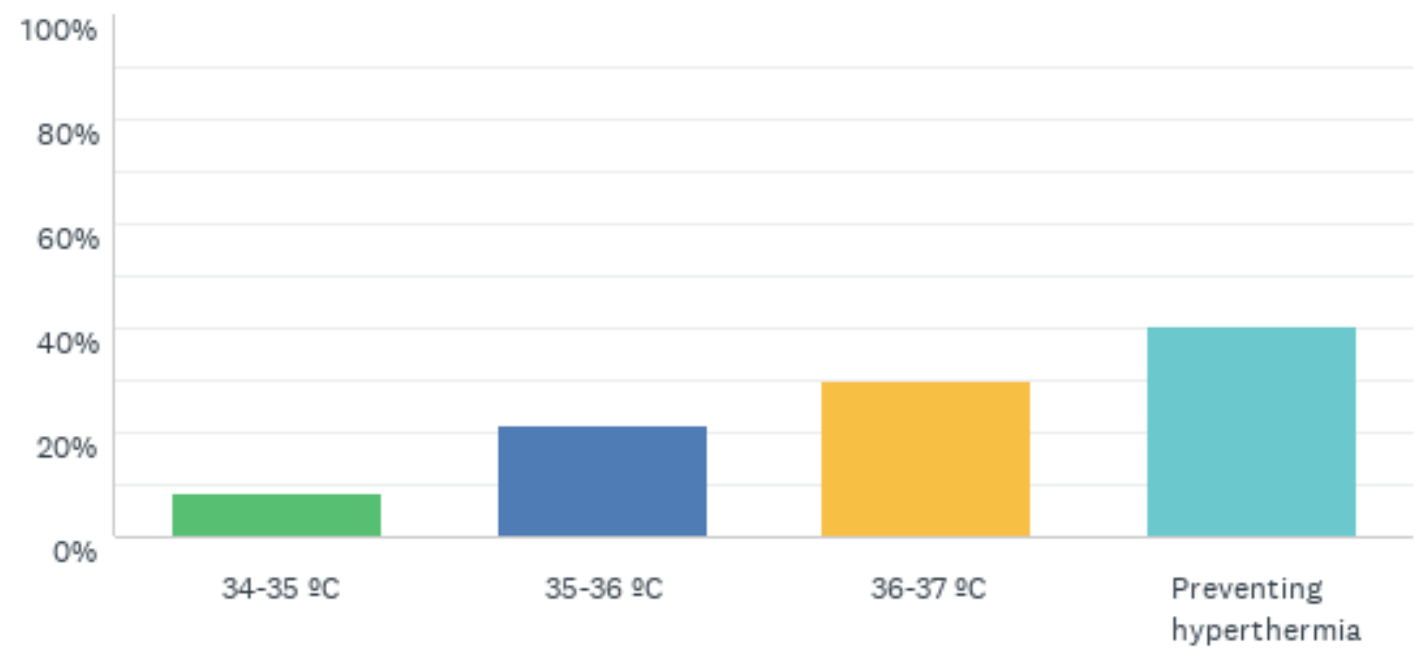

Figure 4

Graphic-4: Target body temperature in pediatric patients with TBI for participant pediatric intensivists 